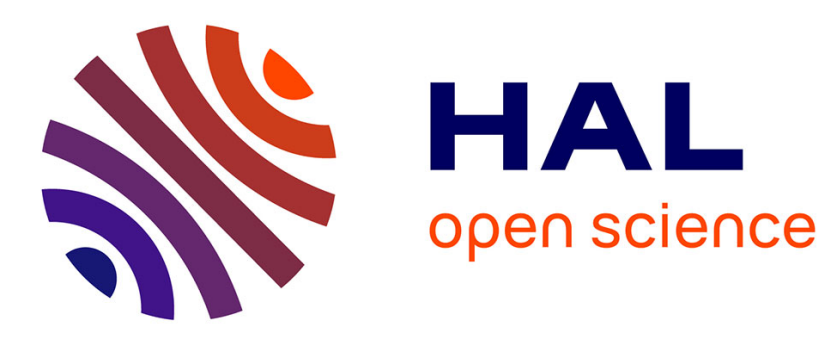

\title{
Optical absorption in degenerate semiconductors
}

\author{
J. Gavoret, P. Nozières, B. Roulet, M. Combescot
}

\section{To cite this version:}

J. Gavoret, P. Nozières, B. Roulet, M. Combescot. Optical absorption in degenerate semiconductors. Journal de Physique, 1969, 30 (11-12), pp.987-997. 10.1051/jphys:019690030011-12098700 . jpa00206867

\section{HAL Id: jpa-00206867 https://hal.science/jpa-00206867}

Submitted on 1 Jan 1969

HAL is a multi-disciplinary open access archive for the deposit and dissemination of scientific research documents, whether they are published or not. The documents may come from teaching and research institutions in France or abroad, or from public or private research centers.
L'archive ouverte pluridisciplinaire HAL, est destinée au dépôt et à la diffusion de documents scientifiques de niveau recherche, publiés ou non, émanant des établissements d'enseignement et de recherche français ou étrangers, des laboratoires publics ou privés. 


\title{
OPTIGAL ABSORPTION IN DEGENERATE SEMICONDUCTORS
}

\author{
By J. GAVORET, P. NOZIERES, B. ROULET and M. COMBESCOT, \\ Groupe de Physique des Solides E.N.S. ${ }^{1}$ ), Faculté des Sciences de Paris, 9, quai Saint-Bernard, Paris, $5^{\mathrm{e}}$ (France).
}

(Reçu le 15 septembre 1969.)

\begin{abstract}
Résumé. - L'absorption optique dans les semiconducteurs dopés à gap direct, dans lesquels la bande de conduction peut être considérée comme un système de Fermi dégénéré, est étudiée au voisinage du seuil de Burstein. Le phénomène est traité dans un modèle simple par les méthodes de perturbation en tenant compte de l'interaction dans l'état final entre les électrons de conduction et le trou profond créé par absorption. Une attention particulière est donnée aux processus de type Auger, qui jouent un rôle fondamental dans la détermination du spectre d'absorption. On montre comment on passe continûment d'un spectre étalé par effet Auger à un spectre résonnant, caractéristique d'un trou infiniment lourd. Une raie d'exciton, distincte du seuil, n'existe jamais.
\end{abstract}

\begin{abstract}
The optical absorption in doped direct gap semiconductors in which the conduction band may be considered as a degenerate Fermi system is studied near the Burstein edge. The problem is treated within a simple model by the methods of perturbation theory taking into account the final-state interaction between conduction electrons and the deep hole created by the photon. Particular attention is paid to Auger processes which play a fundamental role in the determination of the absorption spectrum. It is shown in detail how one passes continuously from the usual Auger broadened Burstein edge to the resonant spectrum characteristic of an infinitely heavy hole. In any case, an exciton line, distinct from the threshold, never exists.
\end{abstract}

I. Introduction. - The optical absorption spectra of insulators usually display an exciton resonance below the absorption threshold. This exciton corresponds to a bound state of the conduction electron and valence hole created by the absorption process. Recently, G. D. Mahan [1] considered the possible existence of such an exciton in direct gap semiconductors, in which the conduction band may be considered as a degenerate Fermi system. In such a case the threshold for direct processes (the so-called Burstein [2] edge) is displaced by an amount :

$$
\frac{k_{\mathrm{F}}^{2}}{2 m_{\mathrm{e}}}+\frac{k_{\mathrm{F}}^{2}}{2 m_{\mathrm{h}}}
$$

where $k_{\mathrm{F}}$ is the Fermi momentum and $m_{\mathrm{e}}$ and $m_{\mathrm{h}}$ the electron and hole masses. In his paper, Mahan considered only the final state interaction between the excited electron and hole, the remaining electrons being frozen in the usual Fermi distribution. He found that there existed a bound state below the Burstein edge with binding energy $\Delta$ of the form :

$$
\Delta=\xi_{0} \mathrm{e}^{-1 / g}
$$

$\xi_{0}$ is a cut-off comparable to the Fermi energy and $g$ an appropriately defined coupling constant.

(1) Laboratoire associé au Centre National de la Recherche Scientifique.
Mathematically, such a calculation amounts to summing the so-called ladder diagrams : it is directly equivalent to the old calculation of Cooper in relation to the bound state of particle-pairs [3]. The existence of such a discrete state below the absorption threshold is rather surprising; Mahan himself argued that such an exciton should have a finite lifetime, which he took into account phenomenologically in calculating the actual absorption spectrum.

The exciton broadening is not only due to ordinary impurity or phonon scattering, but also to Auger-type transitions, in which the optical process is accompanied by the excitation of one or several electrons across the Fermi surface. In the latter processes, momentum can be absorbed by the extra electrons at practically no cost in energy : the indirect absorption threshold $\omega_{\mathrm{I}}$ is thus lower than the direct threshold $\omega_{D}$ as shown on figure 1 . The difference corresponds to the recoil energy of a hole with momentum $k_{\mathrm{F}}$. We show in this paper that such Auger processes, which were ignored in Mahan's calculation, deeply alter the nature of the absorption spectrum; when $\omega_{\mathrm{D}}-\omega_{\mathrm{I}}$ is large (small hole mass) the Mahan exciton disappears completely, the threshold at $\omega_{D}$ is just broadened with a tail extending to $\omega_{\mathrm{I}}$. When $\omega_{\mathrm{D}}-\omega_{\mathrm{I}}$ is comparable to Mahan's binding energy $\Delta$, a resonance develops between the two thresholds. An exciton line, distinct from the threshold, never exists. 


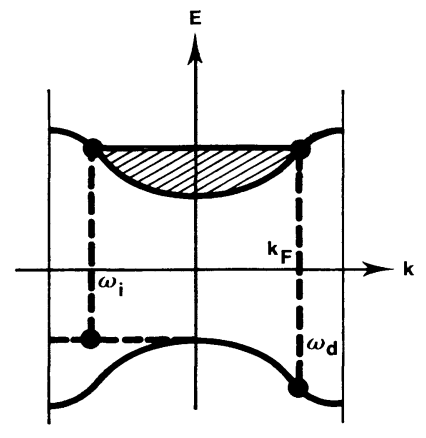

FIG. 1. - Optical absorption in a direct gap semiconductor with a degenerate conduction band. The direct $\omega_{D}$ and indirect $\omega_{\mathrm{I}}$ threshold frequencies.

A similar situation was found in the $\mathrm{X}$ ray absorption of metals $[4,5,6]$. In that case, the hole created in the deep band can be considered as infinitely heavy : the direct and indirect thresholds then coincide. It was shown that the absorption spectrum is singular near $\omega_{\mathrm{D}}$ : in the case of weak electron-hole coupling, to which we shall limit ourselves in this paper, the absorption is infinite at threshold. There again the key role is played by resonant Auger transitions, in which many electrons are excited in the course of the $\mathrm{X}$ ray process. One purpose of this paper is to study in detail how one passes continuously from the usual Auger broadened Burstein edge to the resonant spectrum characteristic of an infinitely heavy hole. Qualitatively we shall see that the $\mathrm{X}$ ray singularity is broadened over the energy range $\omega_{D}-\omega_{I}$ and disappears if that range is too large.

The mathematical formulation of the problem is set up in section II, the respective role of self energy and vertex renormalization is discussed in section III, while section IV deals with the shape of the electron absorption spectrum.

II. Formulation of the problem. - We use a model similar to that introduced in the study of $\mathrm{X}$ ray absorption in metals [4]. The only difference is that the hole now has a finite mass : it is thus necessary to specify its momentum. The hamiltonian of the electron-hole system is written as :

$$
\begin{aligned}
H=\sum_{k} \frac{k^{2}}{2 m_{\mathrm{e}}} a_{k}^{+} a_{k}-\sum_{k}[ & {\left[E_{g}+\frac{k^{2}}{2 m_{\mathrm{h}}}\right] b_{k}^{+} b_{k} } \\
& +\sum_{k, k^{\prime}, q} V_{k k^{\prime}} a_{k}^{+} a_{k^{\prime}} b_{k-q} b_{k^{\prime}-q}^{+}
\end{aligned}
$$

where $a_{k}^{+}$and $b_{k}^{+}$are respectively the creation-operators for conduction and valence electrons, and $E_{g}$ is the direct gap-width. Here again we ignore the Coulomb interaction among conduction electrons, which may be taken into account by introducing suitably renormalized quasiparticles. The interband part of the Coulomb interaction (involving terms such as $a^{+} a^{+} a b$ ) is completely neglected : (3) includes only direct intraband scattering of the electron and the hole.
The coupling to the electromagnetic field is described by a perturbing hamiltonian :

$$
H x=\sum_{k} W_{k} a_{k}^{+} b_{k} \mathrm{e}^{-i \omega t}+h . c
$$

(the momentum of the photon is assumed to be negligible). The optical absorption may be deduced from the response function :

$$
S\left(t-t^{\prime}\right)=\left\langle 0\left|T\left\{H x(t) H x\left(t^{\prime}\right)\right\}\right| 0\right\rangle
$$

which we shall calculate in the framework of perturbation theory. In order to simplify the calculation, we shall use a separable potential :

$V_{k k^{\prime}}=-V u_{k} u_{k^{\prime}} \quad$ with $\quad u_{k}=\left\{\begin{array}{lll}1 & \text { if } & \left|\varepsilon_{k}-\mu\right|<\xi_{0} \\ 0 & \text { if } & \left|\varepsilon_{k}-\mu\right|>\xi_{0}\end{array}\right.$

where $\xi_{0}$ is a cut-off of the order of the Fermi energy. The strength of the interaction is measured by the dimensionless parameter $g=\nu_{0} V$, where $\nu_{0}$ is the density of state at the Fermi level for a given spin; using for $V$ a screened Coulomb interaction averaged over the Fermi surface one finds that [1] :

$$
g \simeq \frac{r_{\mathrm{s}}}{12} \operatorname{Ln}\left(1+\frac{6}{r_{\mathrm{s}}}\right)
$$

where $r_{\mathrm{s}}$ is the usual interparticle spacing measured in Bohr radii. In typical doped semiconductors (Ge or $\mathrm{InSb}) r_{\mathrm{s}} \simeq 1$ which leads to $g \simeq 0.1$ to 0.2 . In the same spirit, we assume that $W_{k}$ does not depend on $k$. In doing such approximations, we loose all information related to the angular symmetry of the various bands; such effects might be included by performing a partial wave analysis of the problem. We shall not attempt to do so, since our main purpose is to study the qualitative shape of the spectrum near the absorption threshold.

Besides the angular dependence of the various factors, we neglect a number of other important physical phenomena; for instance in doped semiconductors we ignore the electron and hole scattering on the impurities. Such an interaction leads to renormalization of the gap and the effective masses [7], which we suppose has been carried out. It also gives rise to tails in the density of states near band extrema, which will act to smear out the spectrum. In particular, for an $n$-type semiconductor, the tail in the valence band broadens the indirect threshold. Fortunately, BonchBruevich [8] has shown that in non compensated materials the tailing effect was mostly important for majority carriers, and relatively small for minority carriers. We can thus reasonably assume that the shape of the valence band is unaffected by impurities $\left({ }^{2}\right)$. The main effect of impurities is to smear

(2) The Burstein edge involves hole-states with momentum of order $k_{\mathrm{F}}$ for which the tailing effect is unessential. Clearly the situation would be quite different if we studied light emission (due to electron-hole recombination) instead of light absorption. In that case the shape of the band near its minimum is all important. 
out electron and hole wave functions in momentum space; as a result, direct transitions no longer conserve momentum. Because of the finite hole mass, such an uncertainty in momentum smears out the direct threshold $\omega_{\mathrm{D}}$ by an amount equal to the uncertainty in the recoil energy of the hole. If $\Gamma=\frac{1}{\tau}$ is the inverse collision time, it is easily found that the threshold smearing is of order $\Gamma \beta$, where $\beta=\frac{m_{\mathrm{e}}}{m_{\mathrm{h}}}$ is the mass ratio. Any structure in the spectrum will be blurred over that range of energies.

Another complication arises when either the conduction or the valence band are anisotropic; in that case the direct threshold $\omega_{D}$ varies over the Fermi surface, which again acts to blurr any structure in the absorption discontinuity. As for impurity scattering, these effects tend to disappear when the hole becomes heavier and heavier. We shall discuss briefly at the end of the paper in which circumstances these effects could become important.

In order to calculate the response function $S\left(t-t^{\prime}\right)$, we introduce the electron and hole Green's functions :

$G\left(k, t-t^{\prime}\right)=-i\left\langle 0\left|T\left\{a_{k}(t) a_{k}^{+}\left(t^{\prime}\right)\right\}\right| 0\right\rangle$

$\mathscr{G}\left(k, t-t^{\prime}\right)=-i\left\langle 0\left|T\left\{b_{k}(t) b_{k}^{+}\left(t^{\prime}\right)\right\}\right| 0\right\rangle$.

The valence band being completely filled in the ground state, $\mathscr{G}\left(k, t-t^{\prime}\right)$ vanishes for $t>t^{\prime}$. The deep hole can thus only propagate in the direction of the increasing times, which bears two important consequences for our problem :

i) The pole of $\mathscr{G}(k, \varepsilon)$, in energy representation, is always to be found in the upper half plane of the complexe variable $\varepsilon$.

ii) The electron propagator $G(k, \varepsilon)$ cannot be renormalized by the electron-hole interaction and has the value :

$$
\begin{aligned}
G(k, \varepsilon) & =G_{0}(k, \varepsilon) \\
& =\frac{1}{\varepsilon-\left(\frac{k^{2}}{2 m_{\mathrm{e}}}-\mu\right)+i \delta \operatorname{sgn}\left(\frac{k^{2}}{2 m_{\mathrm{e}}}-\mu\right)}
\end{aligned}
$$

(in our diagrams, $G$ will be represented by full lines and $\mathscr{G}$ by dashed lines).

We wish to calculate the response function $S(\omega)$, given by all graphs of figure 2 (summed over $\varepsilon, \varepsilon^{\prime}, \mathbf{k}$

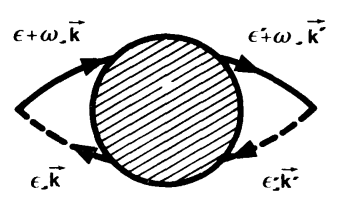

FIG. 2. - A contribution to the response function $\chi(\omega)$. Dashed and full lines refer, respectively, to deep and conduction electron propagators. and $\mathbf{k}^{\prime}$ ). Let us get rid of the dipolar matrix elements by writing the response function $S(\omega)$ in the form :

$$
S(\omega)=|W|^{2} \chi(\omega) .
$$

The simplest approximation for $\chi(\omega)$ is obtained in the previously discussed model of a rigid Fermi sea, which amounts to a summation of the "ladder diagrams" represented on figure 3 . In this model, one

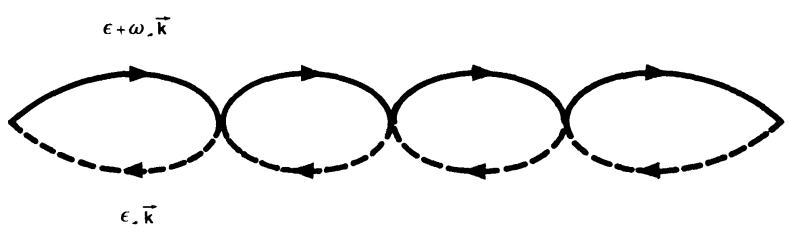

FIG. 3. - A ladder graph contributing to $\chi(\omega)$.

takes into account the sole diffusion between the hole created in the valence band and the electron excited over the Fermi sea into the conduction band. The other electrons do not participate in the diffusion process and are frozen by the exclusion principle. This calculation was performed by Mahan, and yields the following result :

$$
\chi_{\text {Lad }}(\omega)=\frac{\nu_{0} \chi_{0}(\omega)}{1-g \chi_{0}(\omega)}
$$

where $\chi_{0}(\omega)$ is the zeroth order contribution to $\chi(\omega)$ :

$\chi_{0}(\omega)=i \int \frac{\mathrm{d} \varepsilon}{2 \pi} \int \frac{\mathrm{d} \mathbf{k}}{(2 \pi)^{3}} \mathscr{G}_{0}(k, \varepsilon) G_{0}(k, \varepsilon+\omega) u_{k}^{2}$

In order to calculate $\chi_{0}$, we close the integration path in the lower half $\varepsilon$-plane and noticing that :

$$
\mathscr{G}_{0}(k, \varepsilon)=\frac{1}{\varepsilon+\frac{k^{2}}{2 m_{\mathrm{h}}}+E_{f}+\mu-i \delta}
$$

we thus obtain :

$\chi_{0}(\omega)=\int_{k>k_{\mathrm{F}}} \frac{\mathrm{d} \mathbf{k}}{(2 \pi)^{3}} \frac{1}{-\omega+\frac{k^{2}}{2 \nu}+E_{G}-i \delta} u_{k}^{2}$

where $\nu$ is the reduced mass.

$\chi_{0}$ has a branch point for the value :

$$
\omega=\omega_{\mathrm{D}} \equiv E_{G}+\frac{k_{\mathrm{F}}^{2}}{2 \nu} .
$$

In the vicinity of this point :

$$
\begin{aligned}
& \operatorname{Im} \chi_{0}(\omega) \approx \pi v_{0} \frac{\nu}{m_{\mathrm{e}}} \theta\left(\omega-\omega_{\mathrm{D}}\right) \\
& \operatorname{Re} \chi_{0}(\omega) \approx \nu_{0} \frac{\nu}{m_{\mathrm{e}}} \operatorname{Ln}\left|\frac{\xi_{0}}{\omega-\omega_{\mathrm{D}}}\right|
\end{aligned}
$$

where $\theta(\omega)$ is the usual step-function. 
This behaviour is characteristic of the direct transitions : the absorption is discontinuous at the threshold, which leads to a logarithmic divergence of the reactive part of the function $\chi_{0}$. Equation (11) yields the following result for the absorption spectrum :

$\operatorname{Im} \chi_{\operatorname{Lad}}(\omega)=\frac{\nu_{0} \operatorname{Im} \chi_{0}(\omega)}{\left|1-g \operatorname{Re} \chi_{0}(\omega)\right|^{2}+g^{2}\left(\operatorname{Im} \chi_{0}(\omega)\right)^{2}}$.

In this approximation, the continuum of absorption still starts at $\omega=\omega_{\mathrm{D}}$, and an exciton-type bound state appears below the threshold at a distance $\xi_{0} \mathrm{e}^{-1 / g}$ as shown on figure 4 .

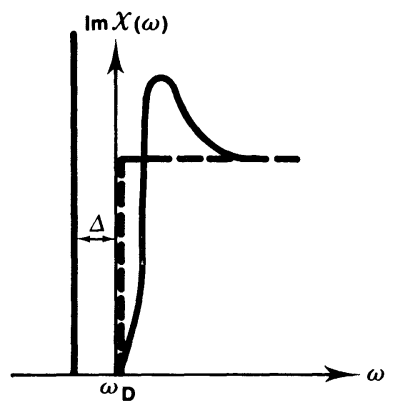

FIG. 4. - Im $\chi(\omega)$ derived in the "ladder approximation". Dotted line refers to zeroth order : $\operatorname{Im} \chi_{0}(\omega)$.

Such a bound state, which exists in insulating semiconductors, has no physical meaning in the degenerate case, where it would be immediately broadened by excitation of other conduction electrons. In order to introduce these processes in the calculation, it is necessary to go beyond the scheme of a rigid Fermi sea, and to allow for the propagation of conduction electrons in both time directions. The simplest corresponding diagrams are shown on figure 5 .

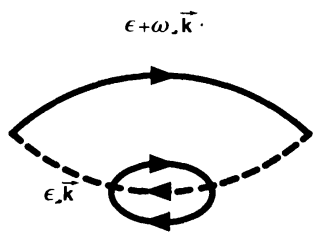

a)

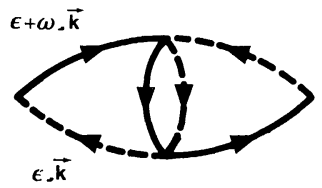

b)
FIG. 5. - The two second order contributions to $\chi(\omega)$ involving indirect transitions :

a) Corresponds to propagator renormalization.

b) Corresponds to vertex renormalization.

It is seen on figure 5 that the intermediate state between the two interaction vertices introduces, in addition to the electron and the deep hole, an excited conduction electron-hole pair. As shown by an elementary calculation, the corresponding absorption is continuous starting from the threshold $\omega_{\mathrm{I}}=E_{G}+\mu$. This behaviour, characteristic of an indirect transition, expresses the fact that the momentum of the inter- mediate hole can take any value. The excited pair carries away the remaining momentum in order to ensure momentum conservation.

In the weak coupling limit and when the two thresholds $\omega_{\mathrm{I}}$ and $\omega_{\mathrm{D}}$ are well apart $(\beta \simeq 1)$, the behaviour of the absorption spectrum close to $\omega_{\mathrm{I}}$ is governed by these two diagrams, which give contributions of the same order $g^{2}$.

This is obviously no longer true in the vicinity of $\omega_{\mathrm{D}}$, where solutions of a Dyson equation for the propagator $\mathscr{G}(k, \varepsilon)$, and of a Bethe-Salpeter equation for the electron hole scattering amplitude, have to be found. In order to take the indirect transitions correctly into account, the deep hole propagator and the electron hole interaction kernel should be renormalized. In part III, the general characteristics of these two types of renormalization are studied and their respective effects on the correlation function are discussed. The response function and the absorption spectrum are effectively calculated in part IV.

III. Renormalization. - Let us first consider the renormalization of $\mathscr{G}$. In the weak coupling limit, the sole diagram involving one excited conduction electron hole pair (shown on fig. 6), should be retained in the calculation of the hole self energy $\Sigma$.

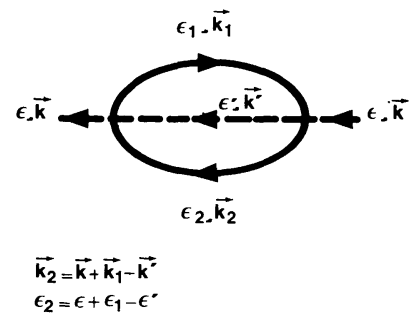

FIG. 6. - The lowest order self energy $\Sigma$ of the deep hole (summed over $\mathbf{k}_{1}, \mathbf{k}^{\prime}, \varepsilon_{1}, \varepsilon^{\prime}$ ).

Let us see the consequences of this renormalization on the elementary bubble of figure 7 where the doubledashed line represents, as usual, the renormalized

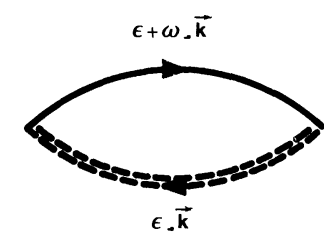

FIG. 7. - The zeroth order contribution $\pi_{0}(\omega)$ to the response function (summed over $\mathbf{k}, \varepsilon$ ). Double dashed line refers to a renormalized deep hole propagator.

propagator. The corresponding contribution can be written similarly to (eq. 14) :

$\pi_{0}(\omega)=\int_{k>k_{\mathrm{F}}} u_{k}^{2} \frac{\mathrm{d} \mathbf{k}}{(2 \pi)^{3}}\left[\mathscr{G}(k, \varepsilon]_{\varepsilon=-\omega+\frac{k^{2}}{2 m_{\mathrm{e}}}-\mu}\right.$. 
The absorption is no longer a step function as it was for $\chi_{0}(\omega)$, but is now controlled by $\operatorname{Im} \Sigma$. It is shown in appendix A that $\operatorname{Im} \pi_{0}(\omega)$ is continuous starting from the indirect absorption threshold $\omega_{I}$. The discontinuity which appeared when the renormalization of the propagator was neglected, is now broadened over an energy range $g^{2} \beta \mu$, where $\beta=\frac{m_{\mathrm{e}}}{m_{\mathrm{h}}}$ is the mass ratio. Although weak, this broadening has important analytical inferences : in particular the corresponding reactive part of the response function is no longer strictly divergent. $R e \pi_{0}$ is a smooth function in $\omega$, with a maximum of order $\operatorname{Ln} \beta g^{2}$ attained to for $\omega \sim \omega_{\mathrm{D}}$.

The summation of the ladder diagrams will be affected by this result, which is a dramatic consequence of the introduction of indirect transitions. Particularly in the case where $g \operatorname{Ln} \beta g^{2} \ll 1$, this summation reduces to its first term, and the absorption is simply given by $\operatorname{Im} \pi_{0}(\omega)$. Any singular behaviour has vanished.

When $g \operatorname{Ln} \beta g^{2} \gtrsim 1$, a solution of the Bethe-Salpeter equation is required. Upon using for the irreducible interaction $I$ the first order vertex, one is directly lead to the summation of ladder diagrams of renormalized elementary bubbles. This amounts to replace $\chi_{0}(\omega)$ by $\pi_{0}(\omega)$ in equation (16). One then finds an absorption spectrum with a double hump near the threshold $\omega_{\mathrm{D}}$, which resembles that found by Mahan. Such a manifestly spurious structure is the outcome of an inconsistent procedure : one must use similar approximations in the self energy and vertex renormalization in order to preserve conservation laws. Indeed, when the renormalization of $I$ is taken into account, this spurious structure vanishes.

In order to remain consistent with our approximation for $\Sigma$, we use for the irreducible interaction $I$ the lowest order diagrams of figure 8 , limiting our study

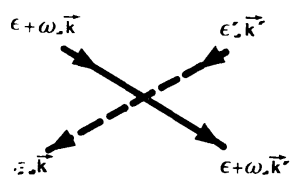

a)

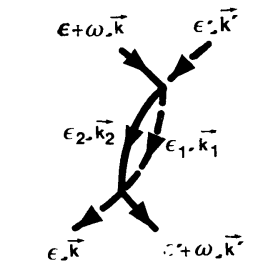

$\epsilon_{1}+\epsilon_{2}=\epsilon+\epsilon^{\circ}+\omega$ $\vec{k}+\overrightarrow{k^{\prime}}=\overrightarrow{k_{1}}+\vec{k}_{2}$

b)
FIG. 8. - The first two diagrams contributing to the irreducible interaction $I_{2}$ (summed over $\varepsilon_{1}, \mathbf{k}_{1}$ ).

to the interaction kernel involved in the response function : the electron and the hole enter the diagram with the same momentum $\mathbf{k}$, and leave with the same momentum $\mathbf{k}^{\prime}$. This diffusion kernel is calculated in appendix B. It should be noticed that $I$ is calculated with a non-renormalized hole propagator. Such a renormalization would merely lead to a negligible correction, in so far as $I$ is not divergent $\left({ }^{3}\right)$. The maximum value reached by its real part is of the order of magnitude of $V g \operatorname{Ln} \beta$.

As soon as the parameter $g \operatorname{Ln} \beta$ becomes of the order of unity, higher order diagrams contributing to $I$ can no longer be neglected. A similar analysis to the one done in the case when $\beta=0$ [4], shows that one must sum up the so-called parquet graphs, built with elementary bubbles of both parallel and antiparallel lines. This calculation is done in the following section.

To conclude, let us recall the different cases which may occur :

1) Strong curvature of the valence band : $g \operatorname{Ln} \beta \ll 1$. In this case, the renormalization of $\mathscr{G}$ renders aimless the summation of the ladder diagrams. The response function is determined by the first renormalized diagram :

$$
\chi(\omega)=\pi_{0}(\omega) .
$$

2) The valence band is almost flat. $g \operatorname{Ln} \beta \gtrsim 1$. The algebra of parquet diagrams is then necessary.

IV. Response function and absorption spectrum. We consider successively the two limiting cases of a light and very heavy hole :

1. $g \operatorname{Ln} \beta \ll 1$ i.e. $\beta \mu \gg \xi_{0} \mathrm{e}^{-1 / g}$. - The distance between the two thresholds is much larger than the binding energy of the "Mahan exciton". Close to $\omega_{\mathrm{D}}$, the absorption is then determined by the sole renormalized elementary diagram of figure 7 , the contribution $\pi_{0}(\omega)$ of which (calculated in appendix A) leads to the absorption spectrum shown on figure $11 \mathrm{a}$. Any structure has been swept away by the indirect transitions, and no trace of an excitonic level remains.

In the immediate vicinity of $\omega_{\mathrm{I}}$, the absorption is controlled by the first two diagrams of the perturbation expansion pictured on figure 5 , and is shown to vary as $g^{2}\left(\omega-\omega_{I}\right)^{7 / 2}$.

2. $g \operatorname{Ln} \beta \gtrsim 1$ i.e. $\beta \mu \lesssim \xi_{0} \mathrm{e}^{-1 / g}$. - The "peak" is then in the vicinity of $\omega_{\mathrm{I}}$, in a region where the continuum is either non existent or still very weak $\left(^{4}\right)$. Every "parquet graph" has then to be taken into

(3) If $\beta=0, I$ is then divergent, but the renormalization of the hole propagator brings about a negligible correction to the absorption spectrum in the weak coupling limit (see ref. [5]).

$\left({ }^{4}\right)$ The position of the exciton peak is shown in Appendix $C$ to be very sensitive to the renormalization of the interaction kernel. Solving the B.S. equation with $I$ but unrenormalized propagators, we find that the peak is all the more nearer to the direct transition threshold $\omega_{D}$ as $\beta$ is smaller. This implies that, in the weak coupling limit, the peak is always to be found in a region where the continuum is already built up. The renormalization of $\mathscr{G}$ then ensures that the peak spreads out into the continuum. Although slightly academic, this calculation clearly displays the decay process of the exciton peak, within the continuum associated with the indirect transitions. 
account. The analysis performed in ref. [4] is briefly recalled and adjusted to the actual problem.

Let $I_{1}$ and $I_{2}$ be the contributions of all irreducible diagrams in respectively the parallel and antiparallel channels. A Bethe-Salpeter equation with the irreducible kernel $I_{2}$ has to be solved. Let us write down this equation for the vertex part $\Lambda(k, \varepsilon, \omega)$, characteristic of the problem we are interested in. The BetheSalpeter equation for $\Lambda$ is represented on figure 9 , the mathematical translation of which being :

$\Lambda(k, \varepsilon ; \omega)=1+\frac{i}{(2 \pi)^{4}} \int \mathrm{d} \mathbf{k}^{\prime} \int \mathrm{d} \varepsilon^{\prime} I_{2}\left(\mathbf{k}, \mathbf{k}^{\prime}, \varepsilon, \varepsilon^{\prime} ; \omega\right)$

$\times G\left(k^{\prime}, \varepsilon^{\prime}+\omega\right) \mathscr{G}\left(k^{\prime}, \varepsilon^{\prime}\right) \Lambda\left(k^{\prime}, \varepsilon^{\prime} ; \omega\right)$.

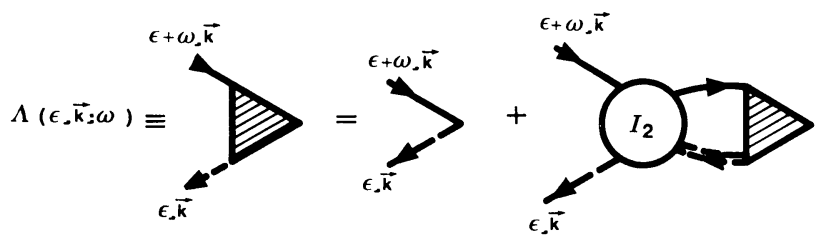

Fig. 9. - The Bethe-Salpeter equation for the vertex part $\Lambda$.

In order to carry out the integration over $\varepsilon^{\prime}$, we note that the hole can only propagate in the direction of increasing times. This ensures that the entrance time $t_{1}$ of a dashed line in a kernel is strictly greater than its exit time $t_{2}$. Similarly, entrance and exit times $\tau$ and $\tau^{\prime}$ of the electron line are necessarily to be found between $t_{1}$ and $t_{2}$, as shown on figure $10 \mathrm{a}$.
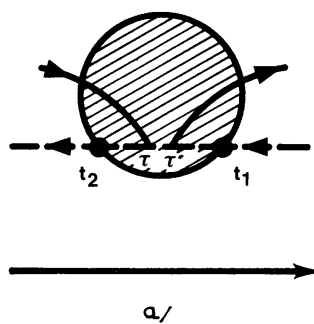

a)

a) Time ordering of the entrance and exit vertices of an interaction kernel.

b) Corresponding energy variables.

In the energy representation (fig. $10 \mathrm{~b}$ ), it follows that the poles of any interaction kernel, e. g. $I_{2}\left(\varepsilon, \varepsilon^{\prime}, \omega\right)$, can only be found in the upper half planes of the complexe variables $\varepsilon$ and $\varepsilon^{\prime}$. A similar conclusion holds for $\mathscr{G}$. The integration over $\varepsilon^{\prime}$ is then easily carried out by closing the contour in the lower half plane. (18) reduces to :

$$
\begin{aligned}
& \Lambda(k, \varepsilon ; \omega)=1+\int_{k^{\prime}>k_{\mathrm{F}}} \frac{\mathrm{d} \mathbf{k}^{\prime}}{(2 \pi)^{3}}\left[I_{2}\left(\mathbf{k}, \mathbf{k}^{\prime}, \varepsilon, \varepsilon^{\prime} ; \omega\right)\right. \\
&\left.\times \mathscr{G}\left(k^{\prime}, \varepsilon^{\prime}\right) \Lambda\left(k^{\prime}, \varepsilon^{\prime} ; \omega\right)\right]_{\varepsilon^{\prime}=-\omega+\frac{k^{\prime 2}}{2 m_{\mathrm{\theta}}}-\mu} .
\end{aligned}
$$

For the same reason the equation for $\chi(\omega)$ :

$\chi(\omega)=\frac{i}{(2 \pi)^{4}} \int \mathrm{d} \varepsilon \int \mathrm{d} \mathbf{k} \Lambda(k, \varepsilon ; \omega) G(k, \varepsilon+\omega) \mathscr{G}(k, \varepsilon)$

reduces to :

$\chi(\omega)=\int_{k>k_{\mathrm{F}}} \frac{\mathrm{d} \mathbf{k}}{(2 \pi)^{3}}[\Lambda(k, \varepsilon ; \omega) \mathscr{G}(k, \varepsilon)]_{\varepsilon=-\omega+\frac{k^{2}}{2 m_{\mathrm{e}}}-\mu}$.

It is thus only necessary to determine $\Lambda$ for the value :

$$
\varepsilon=-\omega+\frac{k^{2}}{2 m_{\mathrm{e}}}-\mu \text {. }
$$

$I_{1}$ and $I_{2}$ are solutions of two coupled integral equations (eq. (20) of ref. [4]). These solutions have been derived for the case where $\beta=0$ and within the framework of a logarithmic approximation. The expression for $I_{2}$ involved in the calculation of the absorption spectrum, was then shown to reduce to the contribution of the sole diagrams of figure 8 . This conclusion still holds for finite values of $\beta$, small enough to let a logarithmic approximation be meaningful. A solution to equation (19) will therefore be looked for upon substituting for $I_{2}$ the expression obtained in appendix B.

An analytical solution may be obtained within the framework of a logarithmic approximation similar to the one previously used [4]. In so far as we are only interested in determining the qualitative feature of the absorption near the resonance, we shall only look for the most singular behaviour of the vertex part $\Lambda$, arising from the logarithmic singularities of the two elementary components $I$ and $\pi_{0}$ in the neighbourhood of the direct threshold $\omega_{\mathrm{D}}$. In this energy region, $I$ can be well approximated by the following simple expression (cf. appendix B) :

$$
I=\left[V+V g \operatorname{Ln}\left\{\max \left(\left|\Omega_{k k^{\prime}}\right|, \beta\right)\right\}\right]
$$

where :

$\Omega_{k k^{\prime}}=\frac{1}{\mu}\left[\omega_{\mathrm{I}}-\omega+\left(\frac{k^{2}}{2 m_{\mathrm{e}}}-\mu\right)+\left(\frac{k^{\prime 2}}{2 m_{\mathrm{e}}}-\mu\right)\right]$.

Substituting everywhere $\left(-\omega+\frac{k^{2}}{2 m_{\mathrm{e}}}-\mu\right)$ for $\varepsilon$ and introducing the notations :

$$
\begin{aligned}
& \xi=\omega_{\mathrm{D}}-\omega+\frac{k^{2}}{2 m_{\mathrm{e}}}-\mu \\
& \xi^{\prime}=\omega_{\mathrm{D}}-\omega+\frac{k^{\prime 2}}{2 m_{\mathrm{e}}}-\mu
\end{aligned}
$$


equation (19) can be written as :

$\Lambda(\xi, \omega)=1+\int_{\omega_{D}-\omega}^{\xi_{0}} \mathrm{~d} \xi^{\prime} I\left(\xi, \xi^{\prime}, \omega\right) \mathscr{G}\left(\xi^{\prime}, \omega\right) \Lambda\left(\xi^{\prime}, \omega\right)$

where :

$$
\begin{aligned}
& I\left(\xi, \xi^{\prime}, \omega\right) \\
& \quad=g+g^{2} \operatorname{Ln}\left\{\max \left(\beta \frac{\left|\xi^{\prime}+\xi+\omega-\omega_{D}-\beta \mu\right|}{\xi_{0}}\right)\right\}
\end{aligned}
$$

and :

$$
\mathscr{G}\left(\xi^{\prime}, \omega\right)=\frac{1}{\xi^{\prime}-\beta\left(\omega_{\mathrm{D}}-\omega\right)-i \Sigma\left(\xi^{\prime}, \omega\right)} .
$$

According to (A.2), the self energy $\Sigma$ itself can be written :

$$
\Sigma\left(\xi^{\prime}, \omega\right)=\frac{2 \pi}{15} \frac{g^{2}}{(\beta \mu)^{3 / 2}}\left[\beta \mu-\xi^{\prime}\right]^{5 / 2} \theta\left(\beta \mu-\xi^{\prime}\right) .
$$

We wish to find a solution to equation (22), taking only into account the most divergent logarithmic terms. In this approximation, the contribution to $\mathscr{G}$ of the self energy becomes important only when $\xi^{\prime} \lesssim g^{2} \beta \mu$. Collecting the most divergent logarithmic terms, we write $(22)$ in the simplified form :

$\Lambda(\xi, \omega)=1+\int_{\max \left(\left|\omega_{D}-\omega\right|, \beta \mu g^{2}\right)}^{\xi_{0}} \frac{\mathrm{d} \xi^{\prime}}{\xi^{\prime}} I\left(\xi, \xi^{\prime}, \omega\right) \Lambda\left(\xi^{\prime}, \omega\right)$.

The lower boundary of the integral in (23) enters through its logarithm. In the range under consideration, in which $g \operatorname{Ln} \beta \sim 1, g \ll 1$, we may replace $\operatorname{Ln}\left(\beta \mu g^{2}\right)$ by $\operatorname{Ln} \beta \mu$, with a logarithmically small error, and then modify accordingly the boundaries of (23). The same approximation may be applied to $\chi$, given by $(20)$, which becomes :

$$
\chi(\omega)=v_{0} \int_{\max \left(\left|\omega-\omega_{\mathrm{D}}\right|, \beta \mu\right)}^{\xi_{0}} \frac{\mathrm{d} \xi}{\xi} \Lambda(\omega, \xi) .
$$

Equations (23) and (24) are then formally similar to those found in ref. [4], except that $\left|\omega-\omega_{D}\right|$ is replaced by $\max \left(\left|\omega-\omega_{1}\right|, \beta \mu\right)$. The same logarithmic approximation may be used, and yields : $\chi(\omega)$

$=\frac{\nu_{0}}{2 g}\left[\exp \left\{-2 g \operatorname{Ln}\left[\frac{1}{\xi_{0}} \max \left(\left|\omega-\omega_{\mathrm{D}}\right|, \beta \mu\right)\right]\right\}-1\right]$

which leads to :

$\chi(\omega)=\frac{\nu_{0}}{2 g}\left[\left(\frac{\xi_{0}}{\max \left(\left|\omega-\omega_{\mathrm{D}}\right|, \beta \mu\right)}\right)^{2 g}-1\right]$.

The absorption is determined by $\operatorname{Im} \chi(\omega)$. The easiest way to get it, is to add the relevant imaginary part to the logarithmic term of (25). This logarithm in the exponent is the real part of the one renormalized bubble $\pi_{0}$; the associated imaginary $f(\omega)$ is the spec- trum modified by Auger broadening, but not by the electron hole scattering resonance. Its general behaviour is shown on figure 11 a. Inserting this imaginary part in (25), we find :

$\operatorname{Im} \chi(\omega) \approx \nu_{0}\left(\frac{\xi_{0}}{\max \left(\left|\omega-\omega_{\mathrm{D}}\right|, \beta \mu\right)}\right)^{2 g} f(\omega)$.

Figure 11 displays the main features of the absorption spectra for different values of the parameter $\beta=\frac{m_{\mathrm{e}}}{m_{\mathrm{h}}}$ which characterizes the curvature of the valence band. It is possible to proceed continuously to the limiting case of an infinitely flat band $(\beta=0)$ previously studied in the problem of $\mathrm{X}$ ray absorption in metals. This case corresponds to the last graph of figure 11 .

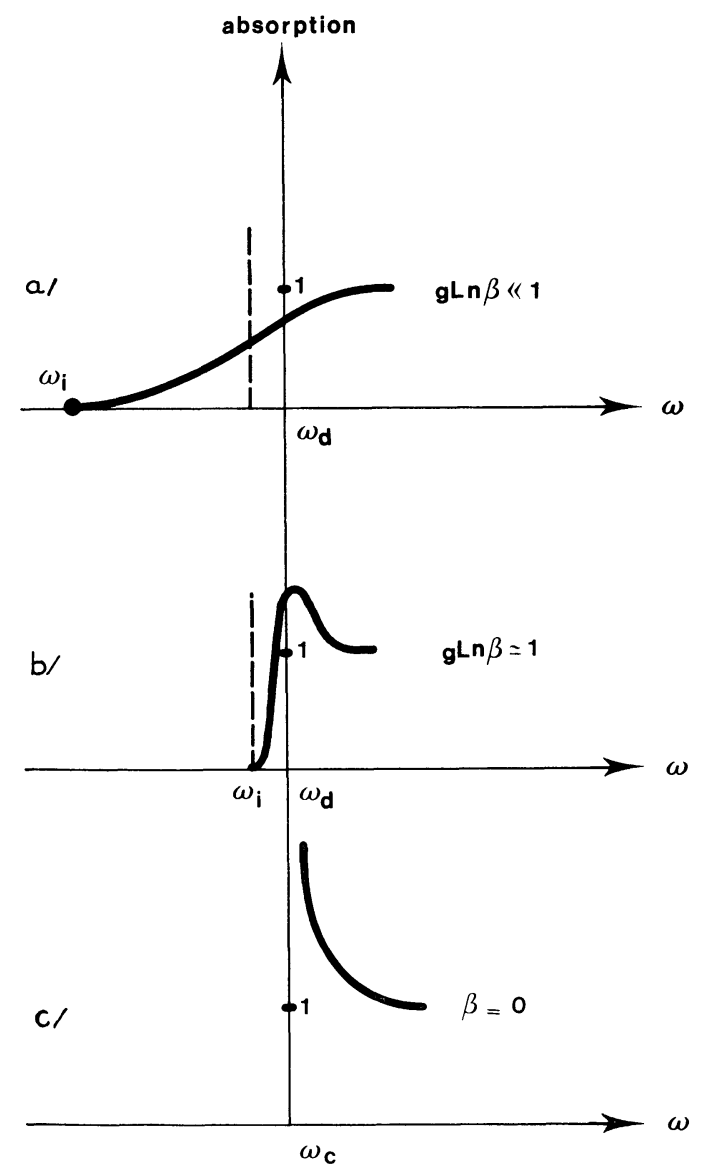

FIG. 11. - The shape of the absorption spectra for different values of the parameter $\beta=\frac{m_{\mathrm{e}}}{m_{\mathrm{h}}}$.

The essential point is to compare the recoil energy of the hole, characterized by the distance between the direct and indirect thresholds, to the binding energy of the bound state obtained while ignoring the indirect transitions. In order to illustrate this discussion, we indicate by a dotted line the position of this unstable bound state. 
Conclusion. - We have shown that whenever the conduction band of a doped semiconductor is filled enough for the electrons to be treated as a degenerate Fermi gas, the exciton level, as observed in the optical absorption of weakly doped semiconductors, vanishes. Indeed, such a bound state is then unstable, and decays by excitation of conduction electron-hole pairs. Taking correctly into account the associated processes, we have displayed the importance of the valence band curvature in the determination of the optical absorption spectra.

We have been able to perform the calculation for different values of the parameter $\beta=\frac{m_{\mathrm{e}}}{m_{\mathrm{h}}}$ and derive the corresponding features of the absorption spectra. When the hole mass increases a resonance develops in the neighbourhood of the absorption threshold, and in the limit where $\beta=0$, it merges into the singularity found in our previous study of metals. The discontinuity observed for an infinite hole mass is spread over a width $\beta \mu$ which is a measure of the hole recoil energy.

In the case of heavily doped semiconductors, the resonance is broadened by other means such as, for instance, scattering of electrons on impurities. If $\Gamma$ measures the corresponding broadening of the spectral density of conduction electrons, it is easily seen that the resonance spreads over a width of order $\Gamma \beta$. In particular when $\beta=0$, the resonance is not affected by impurity scattering; indeed, this resonance is linked to the discontinuity of the Fermi function $n(\varepsilon)$ and not to that of the distribution function of momenta $n(\mathbf{k})$, which alone is modified by scattering on impurities. In so far as $\Gamma$ is very small compared to $\mu$, the result previously obtained can be considered unaltered by the presence of impurities.

Appendix A. - Galculation of the imaginary PART OF THE SELF ENERGY. - To second order in the interaction, the self energy of the deep hole propagator is represented by the diagram (fig. 6). Its contribution is :

$$
\begin{aligned}
\Sigma(\varepsilon, k)=\frac{2 V^{2}}{(2 \pi)^{8}} \iint \mathrm{d} \mathbf{k}^{\prime} \mathrm{d} \mathbf{k}_{1} \mathrm{~d} \varepsilon_{1} \mathrm{~d} \varepsilon^{\prime} \frac{1}{\varepsilon^{\prime}+\frac{k^{\prime 2}}{2 m_{\mathrm{h}}}+E_{G}+\mu-i \delta} \\
\quad \times \frac{1}{\varepsilon_{1}-\left(\frac{k_{1}^{2}}{2 m_{\mathrm{e}}}-\mu\right)+i \delta \operatorname{sign}\left(\frac{k_{1}^{2}}{2 m_{\mathrm{e}}}-\mu\right)} \times \frac{1}{\varepsilon+\varepsilon_{1}-\varepsilon^{\prime}-\left(\frac{k_{2}^{2}}{2 m_{\mathrm{e}}}-\mu\right)+i \delta \operatorname{sign}\left(\frac{k_{2}^{2}}{2 m_{\mathrm{e}}}-\mu\right)} .
\end{aligned}
$$

The integration over the energy variables is straightforward and leads to :

$$
\operatorname{Im} \Sigma(\varepsilon, k)=\frac{2 m_{\mathrm{e}} V^{2}}{(2 \pi)^{5}} \iint_{k_{2}<k_{\mathrm{F}}} \mathrm{k}_{k_{1}} \mathrm{~d} \mathbf{k}^{\prime} \mathrm{d} \mathbf{k}_{1} \delta\left[k_{1}^{2}-k_{2}^{2}+2 m_{\mathrm{e}}\left(\varepsilon+E_{G}+\mu+\frac{k^{\prime 2}}{2 m_{\mathrm{h}}}\right)\right]
$$

the calculation is tedious but not difficult : it can be performed upon using a sequence of transformations into bipolar coordinates. Limiting our study to the expression of $\varepsilon$ involved in the calculation of $\pi_{0}$, $\left(\varepsilon=\frac{k^{2}}{2 m_{\mathrm{e}}}-\mu-\omega\right)$, and for values of $\omega$ within the two thresholds, we find :

$$
\operatorname{Im} \Sigma\left(\frac{k^{2}}{2 m_{\mathrm{e}}}-\mu-\omega, k\right)=\frac{2 \pi}{15} g^{2}\left(\frac{k_{\mathrm{F}}^{2}}{2 m_{\mathrm{e}}}\right)^{-3 / 2}\left[\omega-\omega_{1}-\left(\frac{k^{2}}{2 m_{\mathrm{e}}}-\mu\right)\right]^{5 / 2} \theta\left[\omega-\omega_{\mathrm{I}}-\left(\frac{k^{2}}{2 m_{\mathrm{e}}}-\mu\right)\right] .
$$

The calculation of the "elementary bubble" $\pi_{0}$ with a renormalized propagator ( fig. 7 ) can then easily be performed upon assuming that the small shift in energy associated with $R e \Sigma$ is already included in the definition of the interband gap $E_{G}$ :

$$
\pi_{0}(\omega)=\frac{i}{(2 \pi)^{4}} \iint \mathrm{d} \varepsilon \mathrm{d} \mathbf{k} \frac{1}{\varepsilon+\omega-\left(\frac{k^{2}}{2 m_{\mathrm{e}}}-\mu\right)+i \delta \operatorname{sign}\left(\frac{k^{2}}{2 m_{\mathrm{e}}}-\mu\right)} \times \frac{1}{\varepsilon+E_{G}+\mu+\frac{k^{2}}{2 m_{\mathrm{h}}}-i \operatorname{Im} \Sigma(\varepsilon, k)}
$$


leading to :

$$
\begin{aligned}
& \operatorname{Re} \pi_{0}(\omega)=\frac{1}{(2 \pi)^{3}} \int_{k>k_{\mathrm{F}}} \mathrm{d} \mathbf{k} \frac{-\omega+E_{G}+\frac{k^{2}}{2 \nu}}{\left|-\omega+E_{G}+\frac{k^{2}}{2 \nu}\right|^{2}+\left|\operatorname{Im} \Sigma\left(\varepsilon=-\omega+\frac{k^{2}}{2 m_{\mathrm{e}}}-\mu, k\right)\right|^{2}} \\
& \operatorname{Im} \pi_{\mathbf{0}}(\omega)=\frac{1}{(2 \pi)^{3}} \int_{k>k_{\mathrm{F}}} \mathrm{d} \mathbf{k} \frac{\operatorname{Im} \Sigma\left(\varepsilon=-\omega+\frac{k^{2}}{2 m_{\mathrm{e}}}-\mu, k\right)}{\left|-\omega+E_{G}+\frac{k^{2}}{2 \nu}\right|^{2}+\left|\operatorname{Im} \Sigma\left(\varepsilon=-\omega+\frac{k^{2}}{2 m_{\mathrm{e}}}-\mu, k\right)\right|^{2}} .
\end{aligned}
$$

In so far as we limit our study to values of $\omega$ between $\omega_{\mathrm{I}}$ and $\omega_{\mathrm{D}}$, the most important contribution arises for propagators with momenta close to $k_{\mathrm{F}}$. Thus in the term $\operatorname{Im} \Sigma$ involved in the denominator, $k_{\mathrm{F}}$ is substituded for $k$. The behaviour of $\pi_{0}(\omega)$ is then well described by the following formulae :

$$
\begin{gathered}
\operatorname{Re} \pi_{0}(\omega) \simeq \nu_{0} \operatorname{Ln} \frac{\xi_{0}^{2}}{\left(\omega_{\mathrm{D}}-\omega\right)^{2}+\left(\frac{4 \pi}{30} g^{2} \beta \mu\right)^{2}} \\
\operatorname{Im} \pi_{0}(\omega) \simeq \nu_{0}\left(\frac{\omega-\omega_{\mathrm{I}}}{\beta \mu}\right)^{5 / 2} \operatorname{Arctg}\left(\frac{4 \pi}{30} g^{2} \frac{\omega-\omega_{\mathrm{I}}}{\omega_{\mathrm{D}}-\omega}\right) .
\end{gathered}
$$

Appendix B. - Galgulation of the interaction kernel. - The second order irreducible interaction kernel $J$ is represented by the diagram of the figure $8 \mathrm{~b}$. Its contribution can be written :

$$
\begin{aligned}
J\left(\varepsilon+\varepsilon^{\prime}+\omega, \mathbf{k}, \mathbf{k}^{\prime}\right) & \\
& =\frac{i V^{2}}{(2 \pi)^{4}} \iint \mathrm{d} \mathbf{k}_{1} \mathrm{~d} \varepsilon_{1} \frac{1}{\varepsilon_{1}+\frac{k_{1}^{2}}{2 m_{\mathrm{h}}}+E_{G}+\mu-i \delta} \times \frac{1}{\varepsilon+\varepsilon^{\prime}+\omega-\varepsilon_{1}-\left(\frac{k_{2}^{2}}{2 m_{\mathrm{e}}}-\mu\right)+i \delta \operatorname{sgn}\left(\frac{k_{2}^{2}}{2 m_{\mathrm{e}}}-\mu\right)} .
\end{aligned}
$$

Successive integration over $\varepsilon_{1}$ and transformation into bipolar coordinates lead to :

$$
\operatorname{Re} J(\alpha, q)=-\frac{V^{2}}{(2 \pi)^{2}} \times \frac{1}{q} \int_{0}^{k_{\mathrm{F}}} q \mathrm{~d} q \int_{\left|q-k_{2}\right|}^{q+k_{2}} P \frac{k_{1} \mathrm{~d} k_{1}}{\alpha+\frac{k_{1}^{2}}{2 m_{\mathrm{h}}}-\left(\frac{k_{2}^{2}}{2 m_{\mathrm{e}}}-\mu\right)}
$$

where $q=\left|\mathbf{k}+\mathbf{k}^{\prime}\right|$ and $\alpha=\varepsilon+\varepsilon^{\prime}+\omega+E_{G}+\mu$.

After a straightforward calculation, we get :

$\operatorname{Re} J(\alpha, q)=-\frac{V g}{4} \frac{m_{\mathrm{h}}}{m_{\mathrm{e}}} \frac{k_{\mathrm{F}}}{q}\left\{\left[1-\left(\frac{\gamma+\rho \frac{q}{m_{\mathrm{h}}}}{k_{\mathrm{F}}}\right)^{2}\right] \operatorname{Ln}\left|\frac{k_{\mathrm{F}}-\rho \frac{q}{m_{\mathrm{h}}}-\gamma}{k_{\mathrm{F}}+\rho \frac{q}{m_{\mathrm{h}}}+\gamma}\right|\right.$

$$
\left.-\left[1-\left(\frac{\gamma-\rho \frac{q}{m_{\mathrm{h}}}}{k_{\mathrm{F}}}\right)^{2}\right] \operatorname{Ln}\left|\frac{k_{\mathrm{F}}+\rho \frac{q}{m_{\mathrm{h}}}-\gamma}{k_{\mathrm{F}}-\rho \frac{q}{m_{\mathrm{h}}}+\gamma}\right|-\frac{4 \rho}{m_{\mathrm{h}}} \frac{q}{k_{\mathrm{F}}}\right\}
$$

where $\frac{1}{\rho}=\frac{1}{m_{\mathrm{e}}}-\frac{1}{m_{\mathrm{h}}} \quad$ and $\quad \gamma^{2}=2 \rho\left[\alpha+\mu+\frac{q^{2}}{2\left(m_{\mathrm{h}}-m_{\mathrm{e}}\right)}\right]$. 
The values of $\varepsilon$ and $\varepsilon^{\prime}$ explicitely involved in the calculation of the response function are :

$$
\begin{aligned}
& \varepsilon=-\omega+\frac{k^{2}}{2 m_{\mathrm{e}}}-\mu \\
& \varepsilon^{\prime}=-\omega+\frac{k^{\prime 2}}{2 m_{\mathrm{e}}}-\mu
\end{aligned}
$$

therefore :

$$
\alpha=\mu \Omega_{k k^{\prime}}
$$

with :

$$
\Omega_{k k^{\prime}}=\frac{1}{\mu}\left[\omega_{\mathrm{I}}-\omega+\frac{k^{2}}{2 m_{\mathrm{e}}}-\mu+\frac{k^{\prime 2}}{2 m_{\mathrm{e}}}-\mu\right] .
$$

In so far as $\frac{m_{\mathrm{e}}}{m_{\mathrm{h}}}=\beta \ll 1$ and for values of $\omega$ close to $\omega_{\mathrm{D}}$, an expansion of $R e J$, up to first order in the parameters $\beta$ and $\Omega_{k k^{\prime}}$ can be performed and leads to :

$$
\begin{aligned}
& \operatorname{Re} J\left(\Omega_{k k^{\prime}}, q\right)=\frac{V g k_{\mathrm{F}}}{4 \beta q} \\
& \quad\left\{\left[\Omega_{k k^{\prime}}+\beta\left(1+\frac{q}{k_{\mathrm{F}}}\right)^{2}\right] \operatorname{Ln}\left[\Omega_{k k^{\prime}}+\beta\left(1+\frac{q}{k_{\mathrm{F}}}\right)^{2}\right]\right. \\
& \left.-\left[\Omega_{k k^{\prime}}+\beta\left(1-\frac{q}{k_{\mathrm{F}}}\right)^{2}\right] \operatorname{Ln}\left[\Omega_{k k^{\prime}}+\beta\left(1-\frac{q}{k_{\mathrm{F}}}\right)^{2}\right]\right\} .
\end{aligned}
$$

A study of the different limiting cases then allows one to approximate $R e J$ by the expression :

$$
\operatorname{Re} J\left(\Omega_{k k^{\prime}}, q\right)=V g \operatorname{Ln}\left[\max \left(\left|\Omega_{k k^{\prime}}\right|, \beta\right)\right]
$$

this simplified form of $R e J$ is to be used for the kernel of the Bethe-Salpeter equation.

Appendix C. - In this appendix, a solution of the Bethe-Salpeter equation with the irreducible kernel $I$, but unrenormalized propagators, is calculated within the logarithmic approximation.

This equation, written for the vertex part, is :

$$
\begin{aligned}
& \Lambda(\omega, \xi)=1+\int_{\left|\omega-\omega_{\mathrm{D}}\right|}^{\xi_{0}} \frac{\mathrm{d} \xi^{\prime}}{\xi^{\prime}} \\
& \quad\left[g+g^{2} \operatorname{Ln}\left\{\max \left(\frac{\left|\xi+\xi^{\prime}-\beta \mu\right|}{\xi_{0}} \beta\right)\right\}\right] \Lambda(\omega, \xi) .
\end{aligned}
$$

In so far as we ultimately determine the response function $\chi(\omega)$ which is, within the same approximation, given by :

$$
\chi(\omega)=\nu_{0} \int_{\left|\omega-\omega_{D}\right|}^{\xi_{0}} \frac{d \xi}{\xi} \Lambda(\omega, \xi)
$$

we look for a solution of the Bethe-Salpeter equation only for values of $\xi$ larger than $\left|\omega-\omega_{D}\right|$. A glance at the expression of the integral kernel shows that, when $\left|\omega-\omega_{D}\right|>\beta \mu$, the argument of the logarithm, within the logarithmic approximation, is equal to $\underline{\max \left(\xi, \xi^{\prime}\right)}$

The Bethe-Salpeter equation is then easily solved and yields :

$\chi(\omega)=\frac{\nu_{0}}{2 g}\left[\left|\frac{\xi_{0}}{\omega-\omega_{\mathrm{D}}}\right|^{2 g}-1\right]$ if $\left|\omega-\omega_{\mathrm{D}}\right|>\beta \mu$.

The B.S. equation still has to be solved in the energy region $\left|\omega-\omega_{\mathrm{D}}\right| \lesssim \beta \mu$ where two determinations of $\Lambda(\omega, \xi)$, according to the relative values of $\xi$ and $\beta \mu$, have to be used. These two determinations are solutions of the following system of integral equations :

$\Lambda_{1}(\omega, \xi) \equiv \Lambda(\omega, \xi<\beta \mu)=1$

$$
\begin{aligned}
& +\int_{\left|\omega-\omega_{D}\right|}^{\beta \mu} \frac{\mathrm{d} \xi^{\prime}}{\xi^{\prime}}\left[g+g^{2} \operatorname{Ln} \beta\right] \Lambda_{1}\left(\omega, \xi^{\prime}\right) \\
& +\int_{\beta \mu}^{\xi_{0}} \frac{\mathrm{d} \xi^{\prime}}{\xi^{\prime}}\left[g+g^{2} \operatorname{Ln} \frac{\xi^{\prime}}{\xi_{0}}\right] \Lambda_{2}\left(\omega, \xi^{\prime}\right)
\end{aligned}
$$

$\Lambda_{2}(\omega, \xi) \equiv \Lambda(\omega, \xi>\beta \mu)=1$

$$
\begin{aligned}
& +\int_{\left|\omega-\omega_{\mathrm{D}}\right|}^{\beta \mu} \frac{\mathrm{d} \xi^{\prime}}{\xi^{\prime}}\left[g+g^{2} \operatorname{Ln} \frac{\xi}{\xi_{0}}\right] \Lambda_{1}\left(\omega, \xi^{\prime}\right) \\
& +\int_{\beta \mu}^{\xi} \frac{\mathrm{d} \xi^{\prime}}{\xi^{\prime}}\left[g+g^{2} \operatorname{Ln} \frac{\xi}{\xi_{0}}\right] \Lambda_{2}\left(\omega, \xi^{\prime}\right) \\
& +\int_{\xi}^{\xi_{0}} \frac{\mathrm{d} \xi^{\prime}}{\xi^{\prime}}\left[g+g^{2} \operatorname{Ln} \frac{\xi^{\prime}}{\xi_{0}}\right] \Lambda_{2}\left(\omega, \xi^{\prime}\right) .
\end{aligned}
$$

In order to solve this system, it is convenient to use logarithmic variables. We ultimately find :

$$
\Lambda_{1}(\omega, \xi)=\left(\frac{\xi_{0}}{\beta}\right)^{g}\left[\frac{1}{1-g \operatorname{Ln} \frac{\beta \mu}{\left|\omega-\omega_{\mathrm{D}}\right|}}\right]
$$

$\Lambda_{2}(\omega, \xi)$

$$
=\frac{1}{2}\left\{\left(\frac{\xi_{0}}{\xi}\right)^{g}+\left(\frac{\xi}{\xi_{0}}\right)^{g}\left(\frac{\xi_{0}}{\beta \mu}\right)^{2 g} \frac{1+g \operatorname{Ln} \frac{\beta \mu}{\left|\omega-\omega_{\mathrm{D}}\right|}}{1-g \operatorname{Ln} \frac{\beta \mu}{\left|\omega-\omega_{\mathrm{D}}\right|}}\right\} .
$$

Upon inserting this expression in $\chi(\omega)$ :

$$
\chi(\omega)=\frac{\nu_{0}}{2 g}\left|\left(\frac{\xi_{0}}{\beta \mu}\right)^{2 g} \frac{1+g \operatorname{Ln} \frac{\beta \mu}{\left|\omega-\omega_{D}\right|}}{1-g \operatorname{Ln} \frac{\beta \mu}{\left|\omega-\omega_{D}\right|}}-1\right|
$$

which is valid if $\left|\omega-\omega_{D}\right| \lesssim \beta \mu$.

This result might suggest that the only effect of the renormalization of the interaction kernel is to shift 
the binding energy of the Mahan's exciton from $\xi_{0} \mathrm{e}^{-1 / g}$ to a new value $\beta$ times smaller. Actually this is not the case as much before $\left|\omega-\omega_{\mathrm{D}}\right|$ reaches such a small value, the renormalization of $\mathscr{G}$ has to be taken into account.

When $\left|\omega-\omega_{\mathrm{D}}\right| \lesssim \beta \mu g^{2}$ a little thinking shows that the renormalization of the propagator of the deep hole can be included in the B.S. equation by introducing a cut off or order $\beta \mu g^{2}$ on the first integrals. This is equivalent to substituting $\max \left(\left|\omega-\omega_{\mathrm{D}}\right|, \beta \mu g^{2}\right)$ for the lower boundary $\left|\omega-\omega_{\mathrm{D}}\right|$. Within the energy region we are interested in, the result is thus obtained by substituting $\beta \mu g^{2}$ for $\left|\omega-\omega_{D}\right|$; we get :

$$
\chi(\omega)=\frac{\nu_{0}}{2 g}\left[\left(\frac{\xi_{0}}{\beta \mu}\right)^{2 g} \frac{1-g \operatorname{Ln} g^{2}}{1+g \operatorname{Ln} g^{2}}-1\right]
$$

if $\left|\omega-\omega_{D}\right|<\beta \mu g^{2}$.

One can check on this expression that any possibility of an excitonic peak is swept away by the renormalization of $\mathscr{G}$.

In the limit where $g \ll 1$ one is lead again to result (26).

\section{REFERENCES}

[1] Mahan (G. D.), Phys. Rev., 1967, 153, 882.

[2] Burstein (E.), Phys. Rev., 1954, 93, 632.

[3] Cooper (L. N.), Phys. Rev., 1956, 104, 1189.

[4] Roule'T (B.), Gavoreit (J.) et Nozik̀res (P.), Phys. Rev., 1969, 178, 1072.

[5] Nozièress (P.), Gavoreit (J.) et Roules (B.), Phys. Rev., 1969, 178, 1084.
[6] Nozières (P.) et DE Dominicis (C. T.), Phys. Rev., 1969, 178, 1097.

[7] Wolff (P. A.), Phys. Rev., 1962, 126, 405.

[8] BONCH-BRUEVICH, Electronic theory of heavily doped semiconductors, Elsevier Publ., 1966. 